\title{
ADAPTABLE THRESHOLD ENERGY SCHEME FOR LOAD BALANCING TO IMPROVE NETWORK LONGEVITY IN WIRELESS SENSOR NETWORKS
}

\author{
R. Sumathi ${ }^{1)}$, R. Srinivasan ${ }^{2)}$ \\ ${ }^{1)}$ Siddaganga Institute of Technology, \\ Tumkur, Karnataka, India, rsumathi@sit.ac.in \\ ${ }^{2)}$ RNS Institute of Technology, \\ Bangalore, Karnataka, India, marsri@vsnl.com
}

\begin{abstract}
Energy efficiency is the major concern in the design of Wireless Sensor Network (WSN). It is imperative to develop protocols to distribute energy dissipation across nodes and reduces hotspots in the networks. In this paper, the Load Balancing Dynamic Adaptive Routing (LBDAR) a reactive and efficient data routing protocol is proposed for optimal distribution of load. Rather than finding an optimal path and use it for all communication, the proposed protocol dynamically constitutes energy sufficient routing path from source to sink for each round of communication, by considering two parameters, they are frequency count and adaptable threshold energy. The frequency count provides good chance for all the nodes to actively participate in forwarding data messages and the adaptable threshold energy prevents the early death of heavily involved nodes due to battery drain off, and thus helps in achieving our goal to distribute the load and hence enhances the network lifetime. The performance is evaluated by simulation using $n s-2$ simulator and results show that the LBDAR solves effectively the problem of load balancing and is also more efficient in terms of energy consumption and improves network lifetime.
\end{abstract}

Keywords: Wireless Sensor Network (WSN), Load Balancing, Network lifetime, Adaptive routing, Node Specific parameter, Frequency count, Neighbor list, threshold

\section{INTRODUCTION}

Recent improvements in MEMS (Micro-ElectroMechanical Systems) technology have enabled the development of micro sensing devices capable of local processing and wireless communication. A wireless sensor network is composed of a large number of small, low power, inexpensive sensor nodes, which are densely and randomly deployed either inside the area in which a phenomenon is being monitored or very close to it [1]. The individual nodes have limited and non-replenish energy resources. Events sensed by many source nodes near the phenomena of interest is being forwarded to a special node, named sink node, or base station which has relatively powerful computing and communication capabilities. Data packets to be forwarded to the base station use multi-hop routing using other sensor nodes as relays. This is a dynamically self managed network which can perform automatic corrective actions such as auto-recognizing authorized new device into network, re-routing communication when needed and optimizing the overall network performance among other things. Multi hop routing is typically used to reduce the transmit power and, consequently, increase the battery lifetime and decrease the interference between the nodes. With the energy constrained nature of wireless sensors, it is very important to make efficient use of battery power in order to increase the lifetime of network. In particular, most of the energy of sensors is spent for transmission of data packets generated by the sensor or relaying the packets of other sensors. Hence, it is necessary to design efficient routing protocol in wireless sensor network, with a focus on energy efficiency and lifetime of the network to route the data messages from source to sink node.

Shah.et.al [2] defines network survivability is the most important metric for WSN. To extend WSN lifetime, many routing protocols have been proposed with energy awareness being an essential design consideration. These protocols finds optimal paths and then burn the energy of the nodes along those paths, leaving the network with a wide disparity in the energy level of the nodes and finally results in a network partition. However, there are multipath routing mechanisms, in contrast to energy 
optimizing protocols that find single optimal path, maintains and select a new path among multiple paths from source to sink, alternatively based on energy levels of nodes along each path. Even though multipath routing protocols have potential solution to primary metric of interest network survivability, it requires additional maintenance overhead like setting up and maintaining multipath.

Besides maximizing the network lifetime, it is preferable to burn the energy of nodes more evenly, so that the energy health of entire network should be of the same order. By doing so the nodes in the centre of network continue to provide connectivity for longer duration and time to network partition increases.

In this paper, the Load Balancing Dynamic Adaptive Routing protocol (LBDAR) is proposed, which tries to ensure the even depletion of energy across the nodes, with an objective to increase the energy conservation and prolonging the lifetime of sensor nodes.

Moreover, actual drain on a nodes battery power will depend on the total number of packets forwarded by that node. Hence it is difficult to predict the optimal path unless the total size of the packet stream is known during the path setup. Accordingly, the proposed protocol computes energy required to transmit total packet stream and works on constituting the path by selecting suitable intermediate nodes. The selection of next node depends on two important node specific parameters, (nsp), namely, frequency count and adaptable threshold energy. The frequency count prevents selection of only the specific node and thus, provides an opportunity for all relay nodes to actively participate in forwarding data messages. The energy dissipation will be more in heavily involved node and leads to drain off the battery power in those nodes. In order to avoid early depletion of heavily involved nodes and to prolong network lifetime, sensor nodes are made to enter into reduced activity [3] by maintaining residual energy.

The rest of the paper is organized as follows. We describe some related research work in section-2. Section-3 illustrates problem formulation and operation of the LBDAR protocol. The operational results of the proposed protocol are presented in section- 4 and concluding remarks are discussed in section-5.

\section{RELATED WORK}

Wireless Sensor Networks have been an active research in recent years. The various applications of sensor networks include military, Environmental monitoring, Agriculture, Transportation control, and so on. In many applications the sensors perform measurements of specific parameters like temperature, pressure, movements or other physical values in a periodic or non periodic way. The primary concern is the design and development of an energy efficient routing protocol to forward the collected data of all sensors to the base station.

The potential problem in current protocols is that they find the lowest energy path and use that for every communication. However, that is not the best thing to do for network lifetime. Using a low energy path frequently leads to energy depletion of nodes along the path and leads to network partition. To counteract this problem, Shah.et.al,[2] proposed a multipath scheme i.e., multiple paths are found between source and destination, and each path is assigned a probability of being chosen, depending on the energy metric. Every time data is to be sent from source to destination, one of the paths is randomly chosen depending on the probabilities. This means that none of the paths is used all the time, preventing energy depletion. The multipath routing mechanisms allow the establishment of multiple paths between source and destination. Classical multipath routing has been explored for two reasons. The first is load balancing: traffic between a source-destination pair is split across multiple (partially or completely) disjoint paths. The second use of multipath routing is to increase the likelihood of reliable data delivery.

Energy Balancing Multipath Routing (EBMR) [5] is different from the proposed multipath routing protocols [4], in the methods of establishing, selecting and maintaining routing paths. It increases the lifetime of the sensor network and reduces the additional route maintenance overhead due to the different way the route path being selected. The basic idea of EBMR is that instead of using sourceinitiated or destination-initiated route discovery, it is the base station that finds multipath to the source of the data and selects one to use during the communication. Furthermore, the base station dynamically updates the available energy of each node along the path based on the amount of packets being sent and received. The base station then use the updated energy condition to periodically select a new path from multipath.

In [6], a new energy efficient packet forwarding scheme has been proposed to increase the survivability of low-energy networks. The main idea is to balance the energy consumption among the sensor nodes to avoid early depletion of the networks. Using a deterministic criteria this protocol selects the paths that have the higher relation of nodes energy reserves and distance to destination. The conventional protocols of direct transmission, minimum-transmission-energy routing and static clustering may not be optimal for sensor 
networks, hence LEACH[7] (Low-Energy Adaptive Clustering Hierarchy), a clustering-based protocol that utilizes randomized rotation of local cluster base stations (cluster-heads) to evenly distribute the energy load among the sensors in the network.

Another protocol, energy-aware and contextaware routing of sensor data [8] calls for network clustering and assigns a less-energy-constrained gateway node that acts as a centralized network manager. Based on energy usage at every sensor node and changes in the mission and the environment, the gateway sets routes for sensor data, monitors latency throughout the cluster, and arbitrates medium access among sensors. Directed Diffusion [9] is an important milestone in the datacentric routing research of sensor networks. The idea aims at diffusing data through sensor nodes by using a naming scheme for the data. The main reason behind using such a scheme is to get rid of unnecessary operations of network layer routing in order to save energy.

Chang et al. [10] proposes a flow redirection algorithm which balances the energy consumption rates among the nodes in proportion to the energy available. The objective of this scheme is to maximize the lifetime of the system instead of minimizing the consumed power. The routing protocol [11] aggregates packet streams in a robust way, resulting in energy reductions of a factor 2 to 3.And also more uniform resource utilization is achieved by shaping the traffic flow. The PBMR [12] enables balanced power consumption in a network by managing the routing reply time. The route construction mechanism considers energy usage, and, as a result, it attains balanced energy consumption.

\section{PROBLEM FORMULATION AND DEFINITION}

\subsection{WSN MODEL}

Consider a WSN, modeled as graph $\mathrm{G}=\{\mathrm{N}, \mathrm{D}\}$ shown in figure 1 , comprising a set of sensor nodes $\mathrm{N}$ and together with set $\mathrm{D}$ of edges. The node set $\mathrm{N}$ consists of single base station ' $b$ ', set of sensor nodes $n_{i}=\{i=1, \ldots, m\}$ and set of source nodes $S_{i}=\{i=1, \ldots, l\}$. Each sensor nodes $n_{i}=\{i=1, \ldots m\}$ is associated with a initial amount of battery energy $E_{\text {init }}>0$ and is shown in figure 1. The sink node or the base station ' $b$ ' has unlimited amount of battery energy, memory capacity and computation capabilities. In edge set $D$, there is a edge $d_{(i, j)} \in D$ from sensor node $n_{i}$ to sensor node $n_{j}$, if a single hop transmission from $n_{i}$ to $n_{j}$ is possible. In this model an assumption is made that distance $\mathrm{d}_{(\mathrm{i}, \mathrm{j})}$ among neighbor nodes are equal In this model an assumption is made that distance $\mathrm{d}_{(\mathrm{i}, \mathrm{j})}$ among neighbor nodes are equal. Various notations used in this protocol are:

$n_{i}$ - Sensor node, also called intermediate node

$S_{i}$ - Source node i detect the event and store the data

$E_{\text {init }}$ - Initial energy at node $n_{i}$

$f_{i} \quad$ - Size of sensed data at source $S_{i}$

$E_{\text {res }}^{i}$ - Residual battery power at node $n_{i}$

$E_{T x(i)}^{f i}-$ Energy required to transmit data $f_{i}$ at node $n_{i}$

$E_{R x(j)}^{f i}$ - Energy required to receive data $f_{i}$ at node $n_{i}$

A value pair $(\mathrm{x}, \mathrm{y})$ at each node in figure 1 represents residual battery power in joules and initial frequency count at time ' $\mathrm{t}_{1}$ '. $n_{1}$ to $n_{7}$ indicates node identification, the source node $S_{i}$ gives the sensed data and $b$ is the base station whose value pair is $(\infty,-1)$.The proposed protocol computes the path from multiple sources $S_{i}\{i=1, \ldots, l\}$ to a single base station $b$ involving $\mathrm{p}$ number of nodes in each path, where $\mathrm{p} \subset \mathrm{m}$, at each iteration.

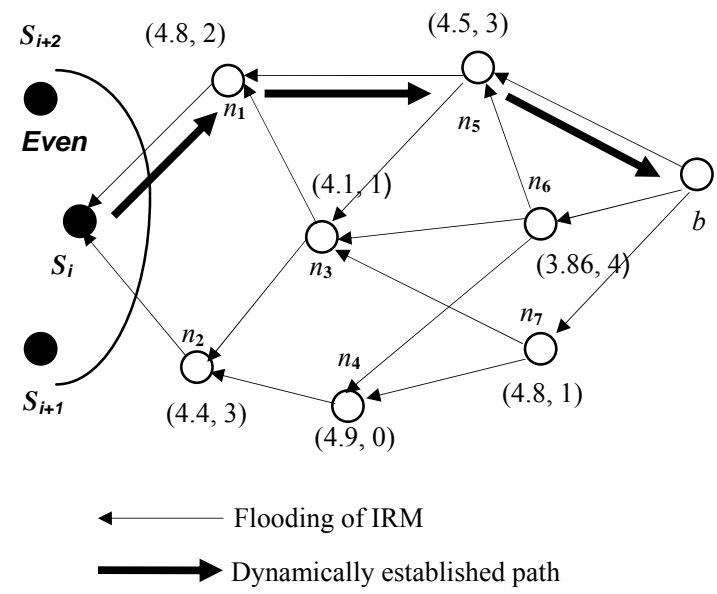

Fig. 1 - Graph of Sensor Nodes illustrating diffusion of IRM along with NSP and establishment of dynamic path

\subsection{DEFINITIONS}

Frequency Count (FC): gives the number of times the node has chosen as a next hop in past communication activities. The count increases by one as it forwards received data message.

Node Specific Parameters (NSP): The information about the node such as residual battery power, frequency count, node_id of the node constitute NSP of the node.

Interest Request Message (IRM): The proposed protocol is a reactive protocol, hence when the data is required form the source nodes, the sink broadcasts request message called IRM towards the source node. The IRM is flooded in the network until the source nodes satisfies the request. The IRM 
carries the NSP of the broadcasting node. The format of IRM packet is given in figure 2 .

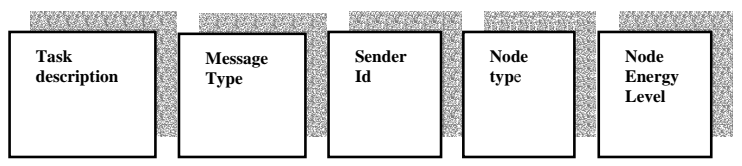

Fig. 2 - Format of Interest Request Message Packet

The field Task description indicates the sensing task. The field Message type indicates that it is an exploratory message. The field Sender Id contains the node id of the message originator. The Node type field indicates whether the originator is a sink, a source or a relay sensor node. The field Energy Level gives the residual battery power of the sending node. The Node frequency count gives the number of times that the node involved in forwarding the data messages.

Neighbor List (NL): The neighbor list of node is a table which maintains the NSP of its downstream nodes.Table-1 gives the neighbor list of node $n_{3}$ from figure 1 .

Table 1: Neighbor list of $\mathbf{n} 3$

\begin{tabular}{|c|r|c|}
\hline Sender Id & $\begin{array}{l}\text { Residual Energy } \\
\text { in joules }\end{array}$ & $\begin{array}{l}\text { Frequency } \\
\text { count }\end{array}$ \\
\hline $\mathrm{n}_{5}$ & 4.10 & 3 \\
\hline $\mathrm{n}_{6}$ & 3.86 & 4 \\
\hline $\mathrm{n}_{7}$ & 4.80 & 1 \\
\hline
\end{tabular}

Adaptable threshold energy $\beta$ : In order to prevent a node from battery drain off an adaptable threshold energy $\beta$ is maintained at each node and it specified by the network administrator. It gives the amount of energy that can be depleted at each node. Once the specified $\beta$ is exhausted, node enters into reduced activity. For example, if $\beta=1 \mathrm{j}$ then, node can be selected a next hop node until $1 \mathrm{j}$ is completely exhausted. Once $\beta$ is set, the usage of energy is limited to

$$
E_{\lim i t}=E_{\text {init }}-\beta
$$

\section{LOAD BALANCING DYNAMIC ADAPTIVE ROUTING PROTOCOL}

\subsection{OVERVIEW}

As soon as the nodes are deployed to an area, where an event is to be monitored, sink node broad casts threshold energy $\beta$, specified by the administrator. Eventually, all sensor nodes compute the $E_{\text {limit }}$ and maintain the same, till it receives the new threshold energy value $\beta$.
The resource rich base station floods the IRM to its neighbors and requests them for information. The interest message describes the sensing task as an attribute value pair [9] and comprises the identifier, residual battery power and frequency count value of the sender. Each node maintains the interest cache and neighbor list. If an incoming message contains fresh interest, then a node creates an interest entry in the cache. For messages regarding the same interest but from distinct senders, the node extracts related information to fill in its neighbor table and resends the interest to its neighbor nodes. Even though the interests have come from a distant sink to its neighbor, this interest appears to be originated from the sending nodes. This process is carried out recursively to ensure that each node will be notified of such interest by doing only local interactions.

When an appropriate node receives an interest, one or more sensors become sources and the LBDAR begins to construct energy sufficient path from initiated source to sink. The LBDAR uses its neighbor list to select and constitute nodes of a path. The LBDAR selects the next node having lesser the frequency count and also should remain with residual battery power is equal to $E_{\text {limit }}$ value after forwarding data packets. When path is established, the sink node sends a SEND MESSAGE packet to the initiated source along the path traversed by IRM. Following the reception of send message, the source node, which has initiated path establishment phase begins to transmit data packets along the dynamically established path.

\subsection{PROTOCOL DESCRIPTION}

The protocol has three phases:

\section{Neighbors Status maintenance phase:}

In this phase, whenever base station needs information, IRM packet, similar to interest diffusion in [9], is disseminated throughout the network, as illustrated in figure 1.

Every node upon receiving the IRM packet does the following:

1. Records the task description, if it is fresh, in the interest cache.

2. Store the NSP in the neighbor list.

3. When node receives same interest again, it discards task description and extract only the NSP and stores in the NL by creating new entry.

4. Changes the sender Id of interest to itself and rebroadcast the IRM packet along with its NSP to its neighbor nodes.

At the end of the neighbor status maintenance phase, each node will have NL table updated. When the interest reaches an appropriate region one or more sensors activated, become sources. 


\section{Path Discovery Phase}

The source nodes satisfy the request are activated and initiates path discovery phase. To begin this phase source node computes the energy, $E_{R E Q} f$, where

$$
E_{r e q}(f i)=E_{T x(j)}^{f i}+E_{R x(j)}^{f i}
$$

which is required to forward the sensed data of size $f i$ from node $n_{i}$ to node $n_{j}$ and the LBDAR performs local interaction in the list to select next hop node and computes residual energy at the selected next hop node:

$$
E_{\text {res }}^{j}=E_{\text {init }}-E_{\text {req }}(f i)
$$

If residual energy at the selected node satisfies the following condition:

$$
E_{\text {res }}^{j} \geq E_{\lim i t}
$$

Then, that node is selected as the next best hop node to route the sensed data (total packet stream). In addition, source node unicasts a PATH DISCOVERY packet shown in figure 3 to the selected router.

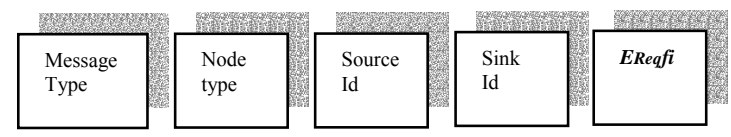

Fig. 3 - Format of PATH DISCOVERY packet

The field message type indicates that it is a path discovery message. The Node type field indicates whether the originator is a sink, a source or a relay sensor node. The field Source Id and Sink Id indicates the node Id of source and sink respectively. Upon the reception of PATH DISCOVERY packet by each selected intermediate node $n_{i}$, the LBDAR algorithm finds the next best optimal node $n_{j}$, using the $n s p$ list of $n_{i}$. The operations of the protocol is given by algorithm- 1 and are repeated at each selected next best optimal node $n_{j}$, till the data packet reaches the sink node. Finally intermediate nodes store the $i d$ of selected next hop node. When PATH DISCOVERY packet reaches sink node, it sends a SEND MESSAGE packet to the initiated source along the selected path.

If none of the nodes in the NL satisfies the condition given in equation-4, it indicates that energy at the nodes reached $E_{\lim i t}$ and nodes are entered into reduced activity. Hence, the sink node begins to set the new value of $\beta$, and thus Einit $=E_{\lim i t}$, and $E_{\lim i t}$ is computed for new threshold value $\beta$ using equation-1.

\section{Algorithm 1: The LBDAR protocol finds the next hop node having sufficient energy}

Input: Neighbor list and path discovery packet.

Output: selects the best optimal next hop node.

Step1: Select a node from NL having least

frequency count value is low.

Step 2: Repeat step 2, until the node i selects the best next hop node j.

Check for residual energy of the selected node even after data transmission.

If $\left(E_{\text {res }}^{j} \geq E_{\lim i t}\right)$ then select the node

Else

select next node having

least frequency count from NL by discarding the selected one.

Step 3: Forward the Path discovery packet to the selected router.

\section{Data Transmission Phase}

In this phase source node begins to transmit data packets along the dynamically established path with the assigned data rates.

Let $p$ number of nodes constitutes a path and involved in forwarding data messages. The residual battery power and frequency count values of $p$ nodes are updated, hence the updated residual battery power of $p$ nodes given by

$$
E_{r e s}^{i}=E_{r e s}^{i}-\left(E_{T x(i)}^{f i}+E_{R x(i)}^{f i}\right) \text { for all } i=1 \text { to } p
$$

and the frequency count value of $p$ nodes increases by

$$
\mathrm{FC}_{i}=\mathrm{FC}_{i}+1 \text { for all } i=1 \text { to } p
$$

This process is repeated for each iteration. Energy levels and frequency count of nodes are updated correspondingly. Eventually, dynamic routing leads to uniform depletion of residual battery power across nodes and helps in enhancing the network lifetime.

\section{RESULTS}

\section{Experimental setup:}

We simulated the protocol using ns-2 simulator. 500 nodes are uniformly deployed in a field of $200 \mathrm{~m}$ $\times 200 \mathrm{~m}$. IEEE 802.11 is used as the MAC layer protocol. The node transmits data packets, which have size of 32 bytes with bandwidth of $200 \mathrm{~kb} / \mathrm{s}$. The transmission range is 40 meters. The whole area is divided into 4 quadrants, sensor nodes are placed at the boundary and sink node is placed at the center $(100,100)$ as shown in figure 4. Initially, energy at all nodes is $5 \mathrm{j}$ and FC is zero. The energy dissipated 
in transmitting and receiving IRM, path discovery and send message packet is not included in the total energy consumption. Base station sends request for data to the sensors in their region of interest, eventually, sensor nodes constructs NL. When interest reaches the appropriate region, the corresponding sensors will initiate path establishment phase followed by data transmission phase.

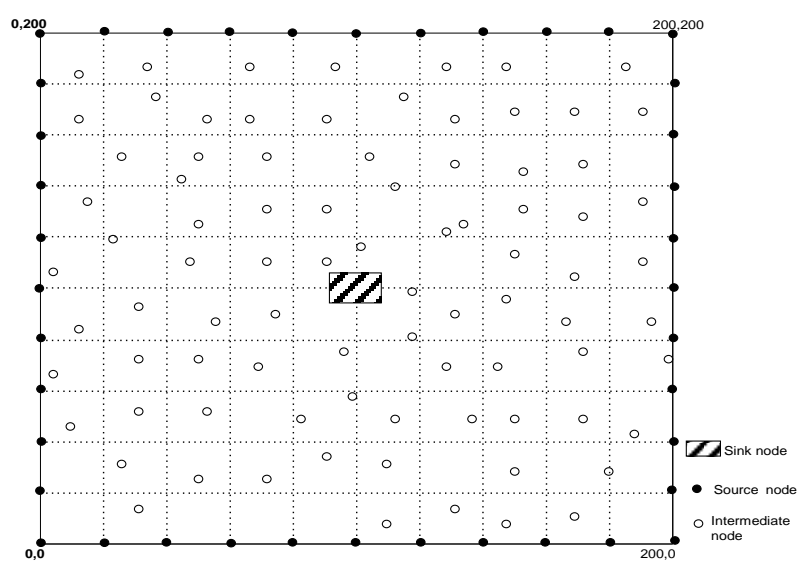

Fig. 4 - Placement of source nodes, Sensor nodes, and Base station on simulation environment

\section{Performance metrics:}

We have compared the proposed protocol with minimum hop routing and energy aware routing against the following measuring metrics of the proposed protocol:

Energy efficiency: This metric is an average of energy consumed by the intermediate nodes and measured at different instance of time during the simulation for the given node density and adaptable threshold values. It indicates the power utilized due to message traffic in the system and depicts the network lifetime.

Time to network partition: When the first node runs out of energy, the network is said to be partitioned.

Standard Deviation of energy consumed: To test energy depletion for different sensors densities we measured standard deviation of energy at different instance of time for various adaptable threshold values by increasing the number of sensors in the system from 100 to 500 with uniform increments.

Packet delivery ratio: It is the ratio of the number of packets successfully arrived at the sink to the total number of data packets sent by source nodes.

Standard deviation of Frequency Count: we calculated standard deviation of frequency count to find the number of times each node participated in forwarding data traffic. Eventually it gives the distribution of load across the nodes.

\section{Performance results:}

Energy efficiency: In order to achieve a longlived network, energy load must be evenly distributed among all sensor nodes so that the energy at a single sensor node or a small set of sensor nodes will not be drained out very soon. Figure 5 shows the average remaining energy at various simulation intervals for intermediate node density $\mathrm{N}=500$. It is obvious that LBDAR can save more energy than minimum hop routing and energy aware routing, especially with time passed and effectively distributes energy consumption evenly among nodes to maximize lifetime of the network. As the time passes, in case of minimum hop routing and energy aware routing data can't be forwarded, even though the energy exist in the network. This is due to energy depletion across the nodes which lies on minimum path and leads to network partition.

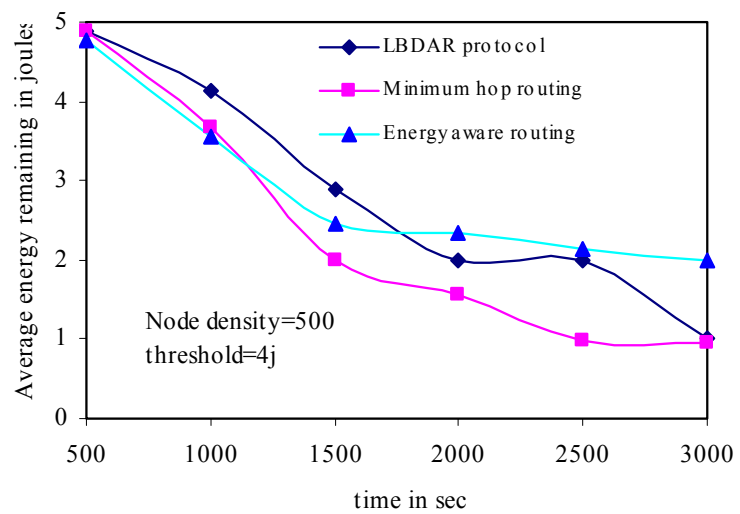

Fig. 5 - Average energy remaining across the nodes

Time to network partition: Figure 6 indicates that time to network portioning in the proposed protocol is greater when compared to other two routing. The Node specific parameter, FC, prevents depletion of energy along optimal path by providing opportunity to all nodes to participate in forwarding of data traffic, and thereby improves the network lifetime.

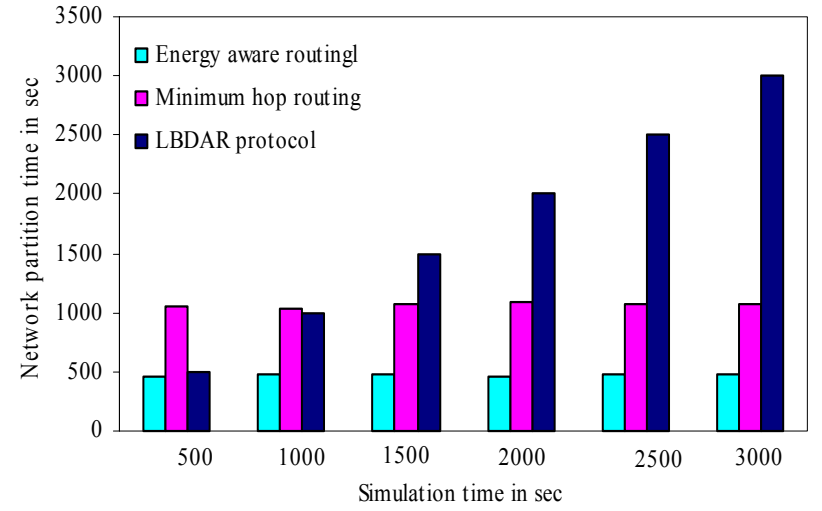

Fig. 6 - Time to network partion for various simulation time 
Whereas in minimum hop routing, continuously minimum path is used for data transmission, hence energy gets depleted along these nodes and the network partition occurs at the earliest time. The results of energy aware routing have indicated that traffic is routed along the paths which are computed based on the distance and energy level. Eventually, energy gets depleted along the optimal path and leads to network partition. The proposed protocol routes the traffic such that the energy consumption is balanced among nodes in proportion to their energy status and hence, maximizes the network lifetime.

Standard Deviation of energy consumed: The standard deviation of energy consumed, in figure 7 indicates that the performance of our protocol is constant with increase in density. On the other hand the rising curve of other two routing schemes indicates that the variance of load increases, as more sensors are included in the system. The results demonstrate that uniform energy dissipation across the sensor nodes.

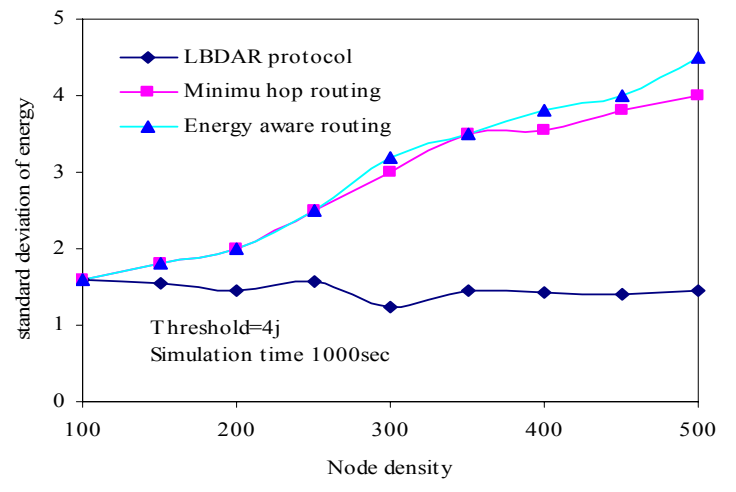

Fig. 7 - Standard deviation of energy for various densities

Packet delivery ratio: Figure 8 shows comparative packet delivery ratio of LBDAR, minimum hop routing and energy aware routing. From figure 8 , it is obvious that nodes die quickly in the beginning and network partition occurs. As a result, packet delivery ratio decreases in case of minimum hop and energy aware routing.

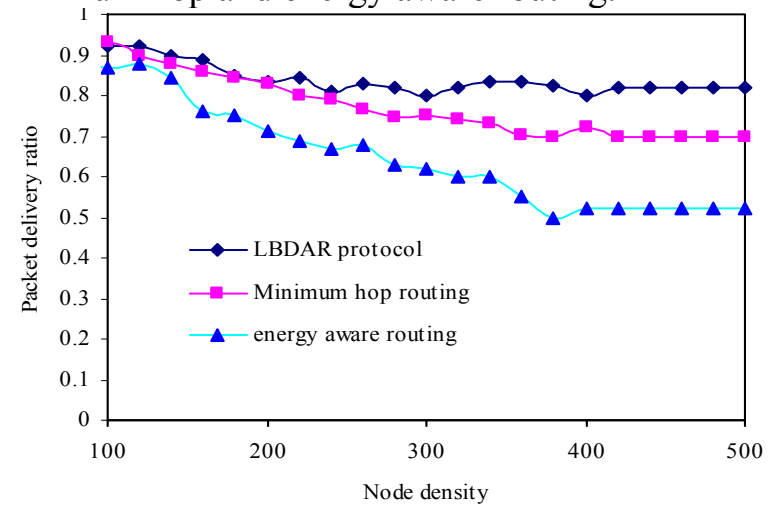

Fig. 8 - Pakcet delivery ratio under various node densities
Standard deviation of frequency count: The frequency count gives the number of times nodes involved in forwarding data traffic. Figure 9 depicts that, the variance of frequency count in LBDAR is uniform for various node densities. This illustrates that all the nodes are uniformly involved in data forwarding activities and hence, load is distributed across the nodes. But, the variance of frequency count increases with increase of density in minimum hop and energy aware routing due to construction of optimal and minimum hop paths from source to sink.

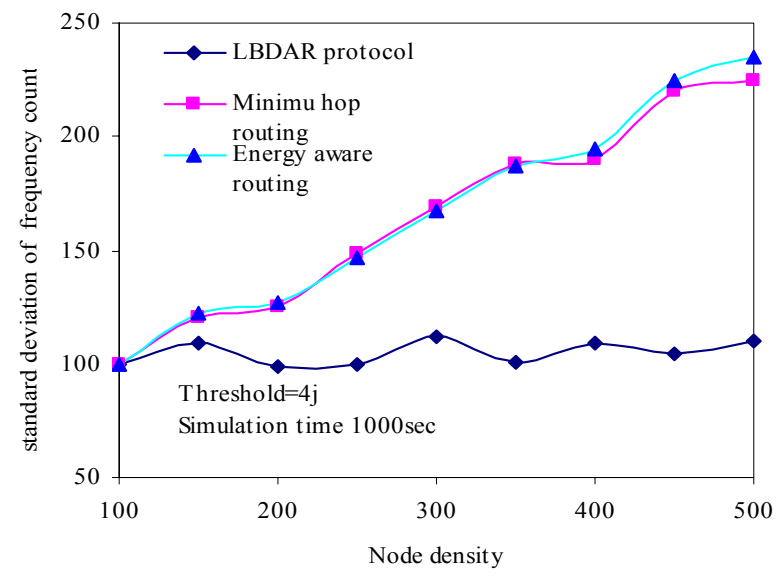

Fig. 9 - Standard deviation of frequency count under various node densities

\section{CONCLUSION}

In this paper, proposed protocol concentrates on two major research issues in WSN. One is load balancing, addressed by a metric, frequency count of node, which plays vital role in selection of suitable next hop node during path set up. In other words it provides an opportunity to all the nodes to actively participate in forwarding data messages and none of the nodes battery power is left without being used. As a result, protocol potentially balances the energy dissipation across the nodes by constituting a path dynamically.

Secondly, enhancing the network lifetime of a WSN is achieved by adaptable threshold energy $\beta$. The ' $\beta$ ' prevents the highly involved node from battery drain off and force the nodes to maintain residual energy and hence, nodes enter into reduced activity.

The results of the proposed protocol demonstrate that more uniform usage of nodes with efficient usage of battery power has been achieved and none of the intermediate nodes have been drained off from battery power, thus enhancing the network lifetime. 


\section{REFERENCES}

[1] I. Akyildiz, W. Su, Y. Sankarasubramaniam, and E. Cayirci. A survey on sensor networks. IEEE Communications Magazine, August 2002, Vol. 40, Issue 8, pp.102-114.

[2] R. C. Shah and J. M. Rabaey. Energy aware routing for low Energy adhoc sensor networks. IEEE Wireless Communications and Networking Conference, Mar. 2002.

[3] A. Hac. Wireless Sensor Network Designs. 2003 John Wiley \& Sons, Ltd, ISBN: 0-47086736-1.

[4] D. Ganesan, R. Govindan, S. Shenker and D. Estrin. Highly-resilient, energy-efficient multipath routing in wireless sensor networks. ACM Mobile Computing and Communications Review, October 2001, Vol. 5, No. 4, P10-24.

[5] Yunfeng Chen, Nidal Nasser. Energy-balancing multipath routing protocol for wireless microsensor networks. QShine'06, Aug.7-9, 2006

[6] Ingrid Teixeira, José Ferreira de Rezende and Aloysio de Castro Pedroza. Wireless sensor network: improving the network energy consumption.

[7] W. R. Heinzelman, A. Chandrakasan, and H. Balakrishnan. Energy efficient communication protocol for wireles microsensor networks. Proceedings of the 33rd IEEE Hawaii International Conference on System Sciences (HICSS), Jan. 2000, pp. 1-10.

[8] M. Younis, M. Youssef, K. Arisha. Energyaware routing in cluster-based sensor networks. in the Proceedings of the 10th IEEE/ACM International Symposium on Modeling, Analysis and Simulation of Computer and Telecommunication Systems (MASCOTS'2002), Fort Worth, Texas, October 2002.

[9] C. Intanagonwiwat, R. Govindan, and D. Estrin. Directed iffusion: a scalable and robust communication paradigm for sensor networks. Proceedings of ACM MobiCom'00, Boston, MA, Aug. 2000, pp. 56-67.

[10] J. H. Chang and L. Tassiulas. Energy conserving routing in wireless ad hoc networks. Proceedings IEEE INFOCOM, Mar. 2000.

[11] C. Schurgers and M. B. Srivastava. Energy efficient routing in wireless Sensor network. ACM Transactions on Embedded Computing
Systems.

[12] Dongshin Kim, Wonjun Lee, Bok-Nyong Park. A power balanced multipath routing protocol in wireless ad-hoc sensor network. Proceedings of The Sixth IEEE international Conference on Computer and Information Technology (CIT'06).

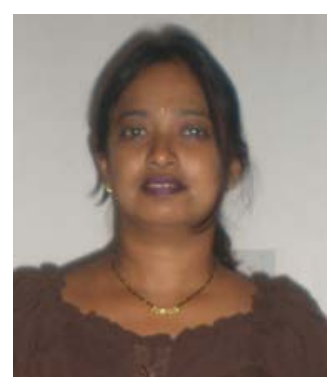

R. SUMATHI received her B.E. and M.E degree in Computer Science and Engineering from Bangalore University, India. She is currently pursuing Ph.D in Department of computer science and engineering at Dr.M.G.R. Educational and Research institute, Chennai, India. Her research interests are routing in wireless sensor networks, Adhoc networks and distributed computing. She authored five papers and published in five international conferences.

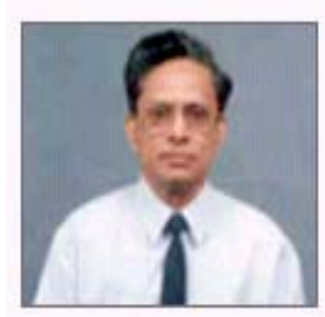

Dr. R. SRINIVASAN received his D.SC from Washington University, St. Louis, USA in 1974 and M.S. from University of Hawaii, USA in 1970. He received his M.Sc degree from Madras University, Chennai

in 1960. Currently working as Professor and Dean for Research and Post graduate studies in Department of Computer Science and Engineering at RNS Institute of Technology, Bangalore, India. He has nearly 45 years of experience in $R \& D$ and Education Sectors, served as Scientist, Computing Activities at National Aeronautical Laboratory, Bangalore, India, for 35 years. After taking voluntary retirement from NAL he served Tata Elxsi, BFL Software and iCMG, as Chief Technology Officer for seven years in all in Software Development. He was the President of Computer Society of India from 1998 to 2000. He is a member of IEEE(USA), Fellow of Computer Society of India, Fellow of IETE, Life member of Instruments Society of India, and Life Member of ISTE. His areas of research are Cryptography and Network Security, Sensor Networks and Distributed Computing. 